\title{
Comparison of 7.0- and 3.0-T MRI and MRA in ischemic-type moyamoya disease: preliminary experience
}

\author{
Xiaofeng Deng, MD, ${ }^{1-4}$ Zihao Zhang, PhD, 5,6 Yan Zhang, MD, PhD, ${ }^{1-4}$ Dong Zhang, MD, PhD, ${ }^{1-4}$ \\ Rong Wang, MD, PhD, ${ }^{1-4}$ Xun Ye, MD, PhD, ${ }^{1-4}$ Long Xu, MD, PhD, ${ }^{1-4}$ Bo Wang, PhD, ${ }^{5}$ Kai Wang, MD, \\ and Jizong Zhao, MD'-4
}

Departments of ${ }^{1}$ Neurosurgery and ${ }^{7}$ Neuroradiology, Beijing Tiantan Hospital, Capital Medical University; ${ }^{2}$ China National Clinical Research Center for Neurological Diseases (NCRC-ND); ${ }^{3}$ Center of Stroke, Beijing Institute for Brain Disorders; ${ }^{4}$ Beijing Key Laboratory of Translational Medicine for Cerebrovascular Disease; ${ }^{5}$ State Key Laboratory of Brain and Cognitive Science, Beijing MRI Center for Brain Research, Institute of Biophysics, Chinese Academy of Sciences; and ${ }^{6}$ Graduate School, University of Chinese Academy of Sciences, Beijing, China

OBJECTIVE The authors compared the image quality and diagnostic sensitivity and specificity of 7.0-T and 3.0-T MRI and time-of-flight (TOF) MR angiography (MRA) in patients with moyamoya disease (MMD).

METHODS MR images of 15 patients with ischemic-type MMD (8 males, 7 females; age 13-48 years) and 13 healthy controls (7 males, 6 females; age 19-28 years) who underwent both 7.0-T and 3.0-T MRI and MRA were studied retrospectively. The main intracranial arteries were assessed by using the modified Houkin's grading system (MRA score). Moyamoya vessels (MMVs) were evaluated by 2 grading systems: the MMV quality score and the MMV area score. Two diagnostic criteria for MMD were used: the T2 criteria, which used flow voids in the basal ganglion on T2-weighted images, and the TOF criteria, which used the high-intensity areas in the basal ganglion on source images from TOF MRA. All data were evaluated by 2 independent readers who were blinded to the strength field and presence or absence of MMD. Using conventional angiography as the gold standard, the sensitivity and specificity of 7.0-T and 3.0-T MRI/MRA in the diagnosis of MMD were calculated. The differences between 7.0-T and 3.0-T MRI and MRA were statistically compared.

RESULTS No significant differences were observed between 7.0-T and 3.0-T MRA in MRA score $(p=0.317)$ or MRA grade $(p=0.317)$. There was a strong correlation between the Suzuki's stage and MRA grade in both 3.0-T $\left(r_{s}=0.930\right.$; $p<0.001)$ and 7.0-T $\left(r_{s}=0.966 ; p<0.001\right)$ MRA. However, MMVs were visualized significantly better on 7.0-T than on 3.0-T MRA, suggested by both the MMV quality score $(p=0.001)$ and the MMV area score $(p=0.001)$. The correlation between the Suzuki's stage and the MMV area score was moderate in 3.0-T MRA $\left(r_{s}=0.738 ; p=0.002\right)$ and strong in 7.0-T MRA $\left(r_{s}=0.908 ; p<0.001\right)$. Moreover, 7.0-T MR images showed a greater capacity for detecting flow voids in the basal ganglion on both T2-weighted MR images $(p<0.001)$ and TOF source images $(p<0.001) ; 7.0-T$ MRA also revealed the subbranches of superficial temporal arteries much better. Receiver operating characteristic curve analysis showed that, according to the T2 criteria, 7.0-T MRI/MRA was more sensitive (sensitivity 1.000; specificity 0.933 ) than 3.0-T MRI/MRA (sensitivity 0.692; specificity 0.933) in diagnosing MMD; based on the TOF criteria, 7.0-T MRI/MRA was more sensitive ( 1.000 vs 0.733 , respectively) and more specific (1.000 vs 0.923 , respectively) than 3.0-T MRI/MRA.

CONCLUSIONS Compared with 3.0-T MRI/MRA, 7.0-T MRI/MRA detected and delineated MMVs more clearly and provided higher diagnostic sensitivity and specificity, although it did not show significant improvement in depicting main intracranial arteries. The authors speculate that 7.0-T MRI/MRA is a promising technique in the diagnosis of MMD because it is noninvasive compared with conventional angiography and it is more sensitive than 3.0-T MRI/MRA.

http://thejns.org/doi/abs/10.3171/2015.5.JNS15767

KEY WORDS moyamoya disease; magnetic resonance imaging; magnetic resonance angiography; $7.0 \mathrm{~T}$; vascular disorders

ABBREVIATIONS $A C A=$ anterior cerebral artery; $A U C=$ area under the curve; $D S A=$ digital subtraction angiography; $I C A=$ internal carotid artery; $I C C=$ interclass correlation coefficient; MCA = middle cerebral artery; MIP = maximum-intensity projection; MMD = moyamoya disease; MMV = moyamoya vessel; MRA = MR angiography; PCA = posterior cerebral artery; PCOA = posterior communicating artery; ROC = receiver operating characteristic; STA = superficial temporal artery; TOF = time of flight. SUBMITTED April 4, 2015. ACCEPTED May 14, 2015.

INCLUDE WHEN CITING Published online November 6, 2015; DOI: 10.3171/2015.5.JNS15767. 
$\mathrm{M}$ OYAMOYA disease (MMD) is an occlusive cerebrovascular disease characterized by stenosis or occlusion at the terminal portion of the bilateral internal carotid arteries (ICAs) and the proximal portion of the middle cerebral arteries (MCAs) and anterior cerebral arteries (ACAs). Compensation for ICA occlusion results in rich arterial collaterals at the base of the brain, the so-called moyamoya vessels (MMVs), which means "puff of smoke" vessels in Japanese. ${ }^{25,30}$ Although its etiology is still uncertain, the disease is found predominantly in Asian populations, which suggests the involvement of hereditary and racial factors. ${ }^{19}$ There are 2 main clinical phenotypes of MMD in Asian populations: the ischemic type, which is common in children, and the hemorrhagic type, which is seen mostly in adults. $3,17,21,32$

Conventional digital subtraction angiography (DSA) has long been considered the gold standard for diagnosing and assessing MMD. However, since the guidelines for diagnosing MMD with MRI and MR angiography (MRA) were published in 1997, time-of-flight (TOF) MRA has been widely accepted as a noninvasive diagnostic modality. When ICA occlusion and MMVs are found with MRA, conventional angiography is unnecessary, particularly in pediatric patients. ${ }^{16}$

The interpretation of high-quality images is crucial when diagnosing MMD with MRI/MRA without conventional DSA. Precise evaluation of abnormal vascular networks in the basal ganglia, in addition to vascular occlusion, is essential for securing a definitive diagnosis of MMD. Nowadays, 3.0-T MR techniques are widely used in clinical situations, and have been reported to show superiority to $1.5-\mathrm{T}$ MR in the diagnosis of MMD.,31 In recent years, however, 7.0-T MRI/MRA has been developed and put into clinical use; it provides a better signal-tonoise ratio and increased $\mathrm{T} 1$ relaxation time than does 3.0T MRI/MRA, which leads to higher-quality MR images, especially in delineating small arteries., ${ }^{41,12}$

To our knowledge, except for one case report, ${ }^{20}$ no other study has focused on comparing 7.0-T and 3.0-T MRI/ MRA in diagnosing MMD. Therefore, the purpose of our study was to systematically compare 7.0-T and 3.0-T MRI and TOF MRA images obtained in patients with ischemictype MMD.

\section{Methods}

All study protocols were approved by the institutional review board of Beijing Tiantan Hospital, Capital Medical University, and written informed consent was obtained from each patient and healthy volunteer. This study enrolled 15 patients with preoperative ischemic-type MMD (MMD group) who were referred to the Department of Neurosurgery at Beijing Tiantan Hospital between January 2013 and July 2014 and 13 healthy controls from the University of Chinese Academy of Sciences (control group). Each subject underwent 3.0-T and 7.0-T MRI and MRA. In addition, every patient with MMD underwent DSA. The main arteries and MMVs and the diagnostic confidence categories were evaluated on 3.0- and 7.0-T MR images separately by 2 independent readers who were blinded to field strength and the presence or absence of MMD.

\section{Group Selection}

The diagnosis of MMD with conventional DSA was based on the guideline reported by Fukui ${ }^{6}$ in 1997: 1) stenosis or occlusion of the terminal ICA and the proximal MCAs and ACAs and 2) bilateral involvement; patients with any other disease that might explain the arterial steno-occlusive disease were excluded. Also excluded were patients with a history of intracranial hemorrhage and patients who had received surgical treatment for MMD. DSA and 3.0-T and 7.0-T MRI/MRA were performed within 2 weeks of each other in all patients, and no clinical event occurred between the 3 examinations.

\section{Healthy Controls}

Thirteen healthy volunteers from the University of Chinese Academy of Sciences without any known cerebrovascular disease were enrolled. Each volunteer underwent 3.0-T and 7.0-T MRI/MRA within 1 week each other.

\section{MRI/MRA Acquisition}

Our study was performed with a 3.0-T Tim Trio (Siemens) scanner and a 7.0-T Magnetom (Siemens) scanner at the Beijing MRI Center for Brain Research, Institute of Biophysics, Chinese Academy of Sciences. The 3.0-T scanner was equipped with a volume coil for transmitting and a 12-channel phased-array head coil for receiving. The following parameters were used for 3.0-T TOF MRA: TR $22.0 \mathrm{msec}$, TE $3.7 \mathrm{msec}$, flip angle $20^{\circ}$, FOV $220 \times 220$ $\mathrm{mm}^{2}$, matrix $384 \times 259$, slice thickness $1 \mathrm{~mm}$, and resolution $0.57 \times 0.85 \times 1.00 \mathrm{~mm}^{3}$. In the 7.0-T Magnetom scanner, a Nova 24-channel phased-array head coil was used for transmitting and receiving, and the parameters were as follows: TR $37 \mathrm{msec}$, TE $5.58 \mathrm{msec}$, flip angle $22^{\circ}$, FOV $200 \times 200 \mathrm{~mm}^{2}$, matrix $768 \times 432$, slice thickness $0.4 \mathrm{~mm}$, and resolution $0.26 \times 0.46 \times 0.40 \mathrm{~mm}^{3}$.

\section{Image Analysis}

Image data were assessed on an OsiriX workstation (version 6.0.1). All images were independently assessed by 1 neuroradiologist and 1 neurosurgeon (each with more than 5 years of experience) who were blinded to field strength and the presence or absence of MMD; discrepancies between the 2 readers were resolved by consensus.

As shown in Table 1, the steno-occlusive severity of intracranial main vessels was evaluated on MRA according to Houkin's grading system (MRA score). ${ }^{14,34}$ Evaluations of the 2 sides of the brain were performed separately, and then only the symptomatic or the more severe hemisphere was chosen for the following analysis. All scores were determined mainly on MRA maximum-intensity projection (MIP) images (Fig. 1), and 3D TOF images were also used referentially. ${ }^{16}$ The MRA score was the sum of the ICA, MCA, ACA, and posterior cerebral artery (PCA) scores. Based on MRA scores, MRA stage grading was defined in 4 grades to conveniently evaluate the progress of the disease: MRA Grade 1, MRA score 0-1; MRA Grade 2, MRA score 2-4; MRA Grade 3, MRA score 5-7; and MRA Grade 4, MRA score 8-10. ${ }^{14,24}$

To assess the MMVs under identical circumstances, we evaluated only transverse MIP images instead of reviewing 
TABLE 1. Houkin's MRA scoring for patients with MMD

\begin{tabular}{|c|c|c|}
\hline Main Artery & Findings & Score \\
\hline \multirow[t]{4}{*}{ ICA } & Normal & 0 \\
\hline & Stenosis of $\mathrm{C}_{1}$ & 1 \\
\hline & Discontinuity of $\mathrm{C}_{1}$ signal & 2 \\
\hline & Invisible & 3 \\
\hline \multirow[t]{4}{*}{ MCA } & Normal & 0 \\
\hline & Stenosis of $M_{1}$ & 1 \\
\hline & Discontinuity of $\mathrm{M}_{1}$ signal & 2 \\
\hline & Invisible & 3 \\
\hline \multirow[t]{3}{*}{ ACA } & Normal $A_{2} \&$ its distal signal & 0 \\
\hline & $\mathrm{A}_{2} \&$ its distal signal decrease or loss & 1 \\
\hline & Invisible & 2 \\
\hline \multirow[t]{3}{*}{ PCA } & Normal $P_{2} \&$ its distal signal & 0 \\
\hline & $\mathrm{P}_{2} \&$ its distal signal decrease or loss & 1 \\
\hline & Invisible & 2 \\
\hline Total & & $0-10$ \\
\hline
\end{tabular}

$A_{2}=$ infracallosal segment of the ACA; $C_{1}=$ knee segment of the ICA; $M_{1}=$ horizontal segment of the MCA; $P_{2}=$ ambient segment of the PCA.

all the angles of the MIP images (Fig. 1), and T2-weighted MR images were also used referentially (Fig. 2A and B). Based on previous studies, we obtained 2 MMV scores to assess the visibility and image quality of the MMVs. According to a study by Jin et al., ${ }^{16}$ the MMV area score was used to evaluate the visibility of MMVs; scores range from 0 to 5 based on the 5 regions, including the basal ganglion, anterior communicating artery, MCA-ICA tip, posterior communicating artery ( $\mathrm{PCoA})-\mathrm{PCA}$, and basilar artery tip areas, where collateral arteries are frequently seen in patients with MMD. The image quality of MMVs was evaluated with MMV quality scores, as follows: 3, excellent (vessel segments are clearly and continuously visualized, and vessel-tissue contrast appears to be high); 2 , visible (vessel segments are visualized and adequate for a confident diagnosis, but vessel-tissue contrast does not appear to be particularly high); 1 , scarcely visible (vessel segments are visualized but inadequate for diagnosis); and 0 , not visible?

According to the guideline, the criteria for diagnosing MMD with MRI and MRA are as follows: 1) stenosis or occlusion at the terminal portion of the ICA and/or at the proximal portion of the ACA and/or the MCA; 2) an abnormal vascular network in the basal ganglia (an abnormal vascular network can be diagnosed when more than 2 flow voids are seen on 1 side of the basal ganglion on MRI); and 3) bilateral appearance of Criteria 1 and 2.9.18 In this study, we used 2 methods to evaluate flow voids in basal ganglia. One traditional way is to count the flow void signals on T2-weighted axial MR images (Fig. 2C and D). Another method is to count the high-signal-intensity areas in basal ganglia on source images from TOF MRA (Fig. 3). ${ }^{23}$ Therefore, 2 criteria were used for diagnosing MMD in this study: the T2 criteria and the TOF criteria. Image selection from 3.0-T and 7.0-T MR data obtained from the same subject was performed simultaneously, because identical section levels could be selected. Regions of
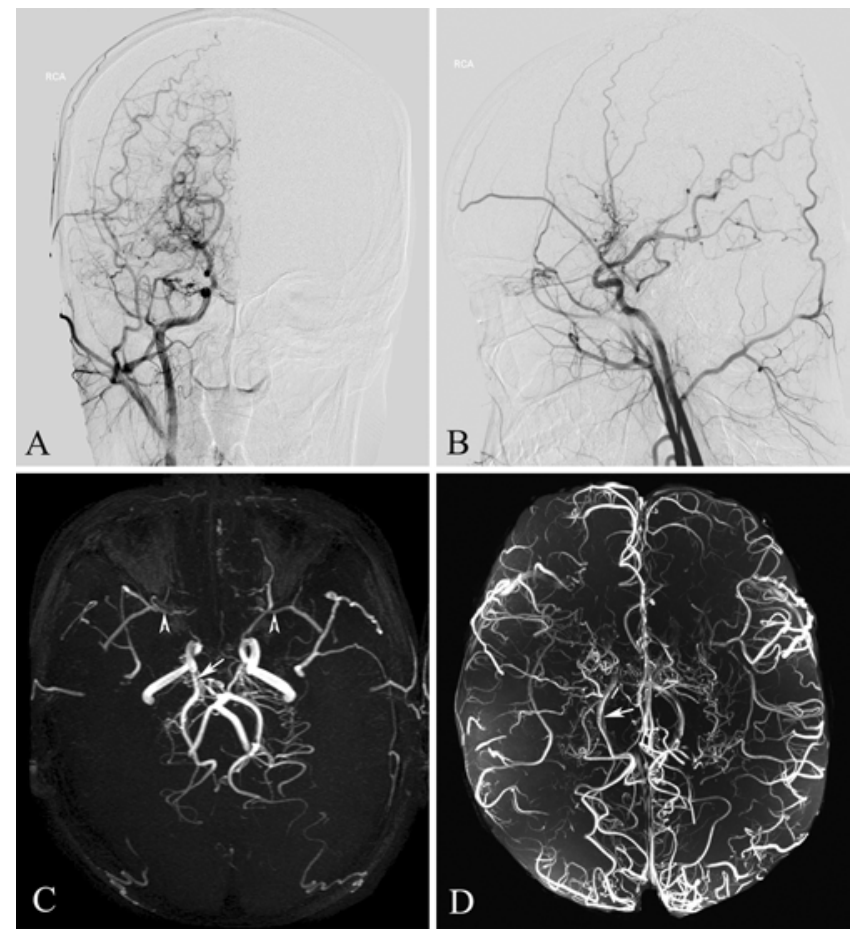

FIG. 1. Patient 7. A and B: DSA images of a patient with MMD. DSA images, anteroposterior and lateral views, respectively, of the right carotid arteries reveal stenosis of the right ICA associated with MMVs, discontinuity of the MCA and ACA, and an enlarged PCoA (Suzuki's Stage III). C: A 3.0-T MRA MIP image showing discontinuity of the right MCA horizontal segment $\left(M_{1}\right.$, arrowheads, MCA score of 2$)$ and disappearance of $\mathrm{A}_{2}$ (ACA score of 2), but the right PCoA was continuous (white arrow, ICA score of 1). MMVs are visible at the right basal ganglion area (MMV area score of 1, MMV quality score of 2). D: A 7.0-T MRA MIP image shows similar main arteries (ICA score 1, MCA score of 2, ACA score of 2), but the visualization of MMVs is better for the ICA-MCA tip, basal ganglion, and PCoA-PCA tip (MMV area score of 3, MMV quality score of 3 ).

interest in the bilateral basal ganglia were selected carefully without including any cisternal structures. According to the Fushimi et al. ${ }^{7}$ study, when counting the highsignal-intensity areas on source images, we determined the threshold for the source images to make the brightest part of brain parenchyma just black out to minimize the variances of the thresholds (Fig. 3C and D). In accordance with a Sawada et al. ${ }^{27}$ study, the confidence of each MMD diagnosis based on MR images was classified into 1 of 5 categories (5, absolutely positive; 4 , probably positive; 3 , unclear; 2 , probably negative; and 1 , absolutely negative).

In addition, MIP images of the superficial temporal artery (STA) in lateral view were also evaluated on both sides for each of the 28 subjects. The main STA, the frontal branch, the parietal branch, and the subbranches were assessed by the 2 observers.

\section{Statistical Analysis}

All statistical analyses were performed with MedCalc Software (Windows version 14.8). MRA scores (including ICA, MCA, and PCA scores), MRA grades, MMV area scores, MMV quality scores, and diagnostic categories according to the $\mathrm{T} 2$ and TOF criteria were compared be- 

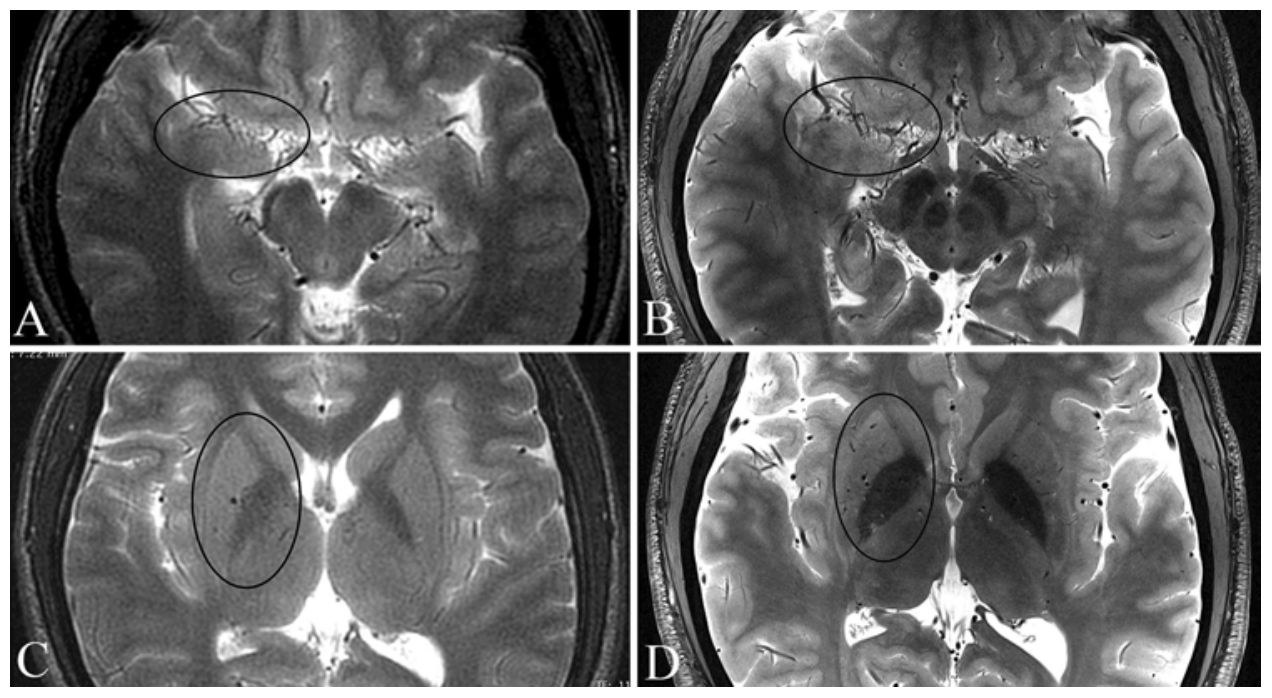

FIG. 2. Patient 7. Axial T2-weighted MR images of a patient with MMD. A and B: 3.0-T and 7.0-T images, respectively, showing MMVs in the sylvian fissure; the 7.0-T MR image is much clearer. C: 3.0-T MR image revealing 1 clear and 4 blurry flow voids in the right basal ganglion. D: 7.0-T MR image revealing 11 flow voids.

tween the 3.0-T and 7.0-T MR images by using the Wilcoxon matched-pair signed-rank test. The paired t-test was performed to compare the number of flow void signals on T2-weighted MR images and the number of high-signalintensity areas on source images from TOF MRA between 3.0-T and 7.0-T MR images, with the left and right sides analyzed independently. Concordance between the 2 observers for 3.0-T and 7.0-T MR images, with respect to the MRA score, MMV area score, MMV quality score, number of flow voids, number of high-signal-intensity areas, and the diagnostic categories, was determined by calculating the interclass correlation coefficient (ICC); an ICC of $>0.75$ indicates good interrater agreement. ${ }^{8}$ The relationship between Suzuki's stages, MRA grades, and
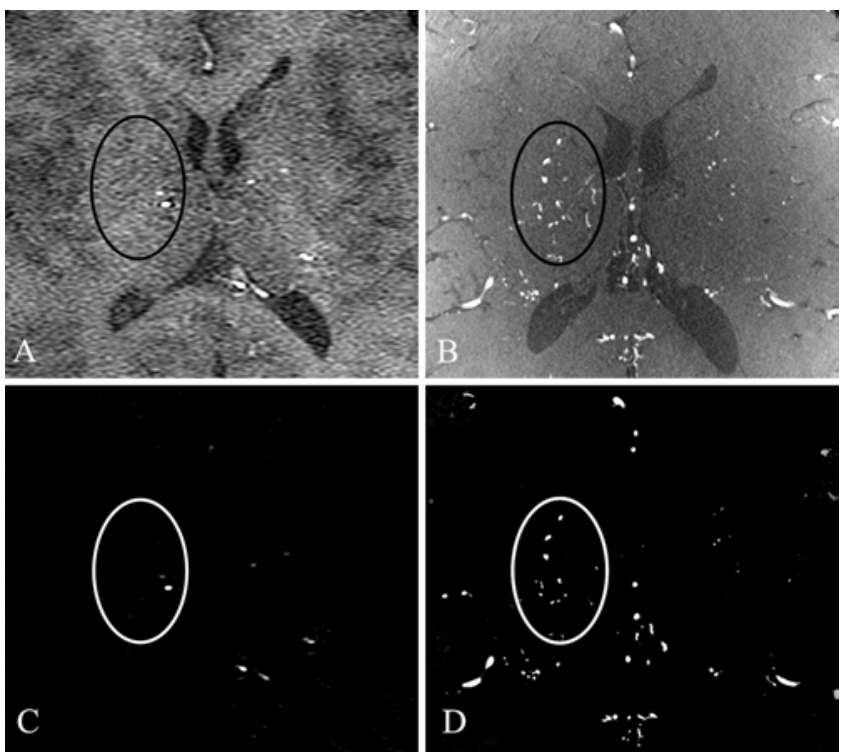

FIG. 3. Patient 7. Source images from TOF MRA of a patient with MMD. 3.0-T MR images ( $\mathrm{A}$ and $\mathrm{C}$ ) showing 2 high-signal-intensity areas, and 7.0-T MR images (B and D) demonstrating 15 high-signal-intensity areas in the right basal ganglion.
MMV area scores was evaluated with Spearman's rank correlation test. A correlation coefficient $\left(\mathrm{r}_{\mathrm{s}}\right)$ of $>0.8$ indicates strong correlation, and an $r_{s}$ of $0.6-0.8$ indicates a moderate correlation. A probability value of $<0.05$ was considered statistically significant.

Regarding the diagnostic accuracy of 3.0-T and 7.0-T MRI/MRA, using DSA as the gold standard, receiver operating characteristic (ROC) curves for both techniques were created for statistical evaluation. The area under the curve (AUC) and the 95\% confidence interval (CI) for each technique were calculated, and comparisons of the AUCs of 3.0-T and 7.0-T MRI/MRA were performed with Hanlay's method. ${ }^{10}$ Moreover, cutoff points were calculated for each technique to maximize sensitivity and specificity. The T2 and TOF criteria were analyzed separately.

\section{Results \\ Population}

For the MMD group, 15 patients with ischemic-type MMD who underwent both 3.0-T and 7.0-T MRI/MRA were enrolled. As shown in Table 2, there were 8 males and 7 females, with ages ranging from 13 to 48 years (mean $29.1 \pm 12.6$ years). Clinical presentations included transient weakness of limbs in 8 patients, headache in 4 patients, language dysfunction in 2 patients, and seizure in 1 patient. According to Suzuki's grading system, there were 5 patients with Stage II, 5 with Stage III, 3 with Stage IV, and 2 with Stage V MMD (Fig. 1A and B).

The control group included 13 healthy volunteers $(7$ males and 6 females) aged 19-28 years (mean $23.2 \pm 2.5$ years). No subject had neurological symptoms.

There were no significant differences between the MMD and control groups in regards to sex $(\mathrm{p}=1.000$, by Fisher's exact test) or age ( $p=0.095$, by independent t-test).

\section{Comparison of MRA Scores Between 3.0-T and 7.0-T MRA}

As shown in Table 2, for the MMD group, the 3.0-T MRA score ranged from 4 to 10 , with 4 patients diagnosed 
TABLE 2. MRA scores and grades of the 15 patients with MMD according to 3.0-T and 7.0-T MRA*

\begin{tabular}{|c|c|c|c|c|c|c|c|c|c|c|c|c|c|c|c|c|}
\hline \multirow[b]{2}{*}{$\begin{array}{l}\text { Pt } \\
\text { No. }\end{array}$} & \multirow[b]{2}{*}{$\begin{array}{l}\text { Age } \\
\text { (yrs) }\end{array}$} & \multirow[b]{2}{*}{ Sex } & \multirow[b]{2}{*}{ Hemisphere } & \multirow[b]{2}{*}{$\begin{array}{l}\text { Suzuki } \\
\text { Stage }\end{array}$} & \multicolumn{6}{|c|}{ 3.0-T MRA Score } & \multicolumn{6}{|c|}{ 7.0-T MRA Score } \\
\hline & & & & & ICA & MCA & ACA & PCA & $\begin{array}{l}\text { MRA } \\
\text { Score }\end{array}$ & $\begin{array}{l}\text { MRA } \\
\text { Grade }\end{array}$ & ICA & MCA & ACA & PCA & $\begin{array}{l}\text { MRA } \\
\text { Score }\end{array}$ & $\begin{array}{l}\text { MRA } \\
\text { Grade }\end{array}$ \\
\hline 1 & 17 & M & $\mathrm{Rt}$ & III & 0 & 3 & 2 & 0 & 5 & 3 & 1 & 3 & 2 & 0 & 6 & 3 \\
\hline 2 & 38 & $\mathrm{~F}$ & $\mathrm{Lt}$ & V & 3 & 3 & 2 & 2 & 10 & 4 & 3 & 3 & 2 & 1 & 9 & 4 \\
\hline 3 & 34 & $\mathrm{~F}$ & Rt & IV & 3 & 3 & 2 & 0 & 8 & 4 & 3 & 3 & 2 & 0 & 8 & 4 \\
\hline 4 & 13 & $F$ & $\mathrm{Lt}$ & III & 1 & 3 & 2 & 0 & 6 & 3 & 1 & 3 & 2 & 0 & 6 & 3 \\
\hline 5 & 44 & M & Rt & II & 1 & 3 & 0 & 0 & 4 & 2 & 1 & 3 & 0 & 0 & 4 & 2 \\
\hline 6 & 13 & M & $\mathrm{Lt}$ & III & 1 & 2 & 1 & 1 & 5 & 3 & 1 & 3 & 1 & 1 & 6 & 3 \\
\hline 7 & 21 & $M$ & $\mathrm{Rt}$ & III & 1 & 2 & 2 & 0 & 5 & 3 & 1 & 2 & 2 & 0 & 5 & 3 \\
\hline 8 & 18 & $F$ & $\mathrm{Lt}$ & IV & 3 & 3 & 1 & 0 & 7 & 3 & 2 & 3 & 2 & 0 & 7 & 3 \\
\hline 9 & 48 & $M$ & $\mathrm{Rt}$ & II & 1 & 3 & 0 & 0 & 4 & 2 & 1 & 3 & 0 & 0 & 4 & 2 \\
\hline 10 & 40 & $\mathrm{M}$ & $\mathrm{Lt}$ & IV & 3 & 3 & 2 & 2 & 10 & 4 & 2 & 2 & 2 & 2 & 8 & 4 \\
\hline 11 & 13 & $F$ & $\mathrm{Rt}$ & II & 1 & 2 & 1 & 0 & 4 & 2 & 1 & 1 & 1 & 0 & 3 & 2 \\
\hline 12 & 36 & $F$ & $\mathrm{Lt}$ & III & 1 & 3 & 1 & 0 & 5 & 3 & 1 & 3 & 1 & 0 & 5 & 3 \\
\hline 13 & 36 & $\mathrm{M}$ & $\mathrm{Rt}$ & II & 1 & 3 & 0 & 0 & 4 & 2 & 1 & 3 & 0 & 0 & 4 & 2 \\
\hline 14 & 42 & $M$ & $\mathrm{Rt}$ & II & 1 & 3 & 1 & 0 & 5 & 3 & 1 & 2 & 1 & 0 & 4 & 2 \\
\hline 15 & 23 & $F$ & $\mathrm{Rt}$ & V & 3 & 3 & 2 & 0 & 8 & 4 & 3 & 3 & 2 & 0 & 8 & 4 \\
\hline
\end{tabular}

$\mathrm{Pt}=$ patient.

* Only the symptomatic or the more severe hemisphere was chosen for analysis.

with MRA Grade 2, 7 patients with Grade 3, and 4 patients with Grade 4 (Fig. 1C); the 7.0-T MRA score ranged from 3 to 9, with 5 patients diagnosed with MRA Grade 2, 6 patients with Grade 3, and 4 patients with Grade 4 (Fig. 1D). According to the results of the Wilcoxon matchedpair signed-rank test, there were no significant differences between 3.0-T and 7.0-T MRA in regard to the ICA (p $=0.564), \operatorname{MCA}(\mathrm{p}=0.317)$, ACA $(\mathrm{p}=0.317)$, PCA $(\mathrm{p}=$ $0.317)$, or MRA ( $p=0.317)$ scores. No significant difference was found in the MRA grade, either $(p=0.317)$.

For the control group, no artery stenosis or occlusion was observed; thus, the MRA score was 0 for all volunteers according to both 3.0-T and 7.0-T MRA.

\section{Comparison of MMV Scores Between 3.0-T and 7.0-T MRA}

For the MMD group, as shown in Table 3, the MMV area score of 3.0-T MRA ranged from 0 to 3 (mean 1.47 \pm 1.06) (Figs. 1C and 2A), and the MMV area score of 7.0-T MRA ranged from 1 to 5 (mean $3.40 \pm 1.40$ ) (Figs. 1D and 2B). A significant difference was found between them ( $\mathrm{p}=$ 0.001 , by Wilcoxon matched-pair signed-rank test). Likewise, the MMV quality score of 7.0-T MRA (mean $2.73 \pm$ $0.46)$ was also significantly higher than that of 3.0-T MRA (mean $1.33 \pm 0.98)(\mathrm{p}=0.001$, by Wilcoxon matched-pair signed-rank test).

For the control group, no MMVs were observed in any case. Therefore, the MMV area score and MMV quality score were both 0 for all the controls.

\section{Comparison of Number of Flow Voids in Basal Ganglia and Diagnostic Categories Between 3.0-T and 7.0-T MR Images}

T2 Criteria

The numbers of flow voids in bilateral basal ganglia on T2-weighted MR images are shown in Table 4. For the MMD group, 0 or 1 flow void was observed in $23(76.7 \%)$ sides on 3.0-T MR images and in $6(20.0 \%)$ sides on 7.0-T MR images. There were significantly more flow voids detected in 7.0-T than in 3.0-T MR images (Fig. 2C and D) on both the left $(\mathrm{p}<0.001$, by paired $\mathrm{t}$-test $)$ and the right $(\mathrm{p}$ $<0.001$, by paired t-test) sides. According to the T2 criteria, the diagnostic categories were significantly higher in 7.0-T than in 3.0-T MR images ( $\mathrm{p}=0.005$, by Wilcoxon matched-pair signed-rank test).

TABLE 3. MMV area and quality scores according to 3.0-T and 7.0-T MRA for patients with MMD*

\begin{tabular}{cccccc}
\hline & \multicolumn{2}{c}{ MMV Area Score } & & \multicolumn{2}{c}{ MMV Quality Score } \\
\cline { 2 - 3 } \cline { 6 - 6 } Pt No. & 3.0-T MRA & 7.0-T MRA & & 3.0 -T MRA & 7.0-T MRA \\
\hline 1 & 2 & 5 & & 1 & 3 \\
\hline 2 & 2 & 5 & 2 & 3 \\
\hline 3 & 1 & 5 & & 1 & 3 \\
\hline 4 & 2 & 3 & & 2 & 3 \\
\hline 5 & 0 & 1 & 0 & 2 \\
\hline 6 & 3 & 4 & 2 & 3 \\
\hline 7 & 1 & 3 & 2 & 3 \\
\hline 8 & 3 & 4 & & 3 & 3 \\
\hline 9 & 0 & 2 & & 0 & 2 \\
\hline 10 & 2 & 5 & 3 & 3 \\
\hline 11 & 0 & 2 & & 0 & 3 \\
\hline 12 & 1 & 3 & & 1 & 3 \\
\hline 13 & 1 & 2 & & 1 & 2 \\
\hline 14 & 1 & 2 & & 1 & 2 \\
\hline 15 & 3 & 5 & 2 & 3 \\
\hline
\end{tabular}

* Only the symptomatic or the more severe hemisphere was chosen for analysis. 
TABLE 4. Flow voids in bilateral basal ganglia on T2-weighted MR images and high-signal-intensity areas on source images from TOF MRA and the diagnostic categories of 3.0-T and 7.0-T MRI/MRA

\begin{tabular}{|c|c|c|c|c|c|c|c|c|c|c|c|c|c|}
\hline \multirow[b]{3}{*}{ Group } & \multirow[b]{3}{*}{ No. } & \multicolumn{4}{|c|}{$\begin{array}{l}\text { No. of Flow Voids on } \\
\text { T2-Weighted MR Images }\end{array}$} & \multicolumn{2}{|c|}{$\begin{array}{l}\text { Diagnostic } \\
\text { Category* }\end{array}$} & \multicolumn{4}{|c|}{$\begin{array}{l}\text { No. of High-Signal-Intensity } \\
\text { Areas on TOF MRA } \\
\text { Source Images }\end{array}$} & \multicolumn{2}{|c|}{$\begin{array}{l}\text { Diagnostic } \\
\text { Category† }\end{array}$} \\
\hline & & \multicolumn{2}{|c|}{ 3.0-T } & \multicolumn{2}{|c|}{$7.0-T$} & \multirow{2}{*}{$\begin{array}{c}3.0-\mathrm{T} \\
\text { MR }\end{array}$} & \multirow{2}{*}{$\begin{array}{c}7.0-T \\
\text { MR }\end{array}$} & \multicolumn{2}{|c|}{ 3.0-T MR } & \multicolumn{2}{|c|}{ 7.0-T MR } & \multirow{2}{*}{$\begin{array}{c}3.0-T \\
\text { MR }\end{array}$} & \multirow{2}{*}{$\begin{array}{c}7.0-T \\
\text { MR }\end{array}$} \\
\hline & & $\mathrm{Lt}$ & $\mathrm{Rt}$ & $\mathrm{Lt}$ & Rt & & & $\mathrm{Lt}$ & Rt & $\mathrm{Lt}$ & Rt & & \\
\hline \multirow[t]{15}{*}{ MMD } & 1 & 0 & 0 & 5 & 4 & 1 & 5 & 3 & 2 & 6 & 10 & 5 & 5 \\
\hline & 2 & 1 & 0 & 3 & 4 & 2 & 5 & 5 & 4 & 26 & 20 & 5 & 5 \\
\hline & 3 & 1 & 1 & 7 & 9 & 3 & 5 & 2 & 1 & 18 & 15 & 4 & 5 \\
\hline & 4 & 0 & 3 & 3 & 4 & 3 & 5 & 3 & 5 & 7 & 13 & 5 & 5 \\
\hline & 5 & 0 & 0 & 3 & 4 & 1 & 5 & 0 & 0 & 3 & 7 & 1 & 5 \\
\hline & 6 & 3 & 3 & 4 & 8 & 5 & 5 & 2 & 4 & 12 & 26 & 5 & 5 \\
\hline & 7 & 0 & 5 & 6 & 11 & 3 & 5 & 2 & 2 & 7 & 15 & 5 & 5 \\
\hline & 8 & 1 & 0 & 7 & 7 & 2 & 5 & 1 & 0 & 12 & 9 & 2 & 5 \\
\hline & 9 & 1 & 0 & 1 & 3 & 2 & 4 & 0 & 1 & 1 & 4 & 2 & 4 \\
\hline & 10 & 3 & 4 & 5 & 6 & 5 & 5 & 5 & 5 & 13 & 21 & 5 & 5 \\
\hline & 11 & 0 & 1 & 2 & 4 & 2 & 5 & 1 & 1 & 7 & 8 & 3 & 5 \\
\hline & 12 & 0 & 0 & 3 & 2 & 1 & 5 & 0 & 0 & 8 & 7 & 1 & 5 \\
\hline & 13 & 0 & 0 & 1 & 1 & 1 & 3 & 0 & 0 & 6 & 5 & 1 & 5 \\
\hline & 14 & 0 & 0 & 0 & 0 & 1 & 1 & 0 & 0 & 4 & 5 & 1 & 5 \\
\hline & 15 & 4 & 1 & 4 & 1 & 4 & 4 & 4 & 2 & 8 & 2 & 5 & 5 \\
\hline \multirow[t]{13}{*}{ Control } & 1 & 0 & 0 & 0 & 0 & 1 & 1 & 0 & 0 & 0 & 0 & 1 & 1 \\
\hline & 2 & 0 & 0 & 0 & 0 & 1 & 1 & 0 & 0 & 1 & 1 & 1 & 1 \\
\hline & 3 & 0 & 0 & 0 & 0 & 1 & 1 & 0 & 0 & 2 & 2 & 1 & 3 \\
\hline & 4 & 0 & 0 & 0 & 0 & 1 & 1 & 0 & 0 & 1 & 0 & 1 & 1 \\
\hline & 5 & 0 & 0 & 0 & 0 & 1 & 1 & 1 & 1 & 1 & 1 & 1 & 1 \\
\hline & 6 & 0 & 0 & 0 & 0 & 1 & 1 & 0 & 0 & 1 & 1 & 1 & 1 \\
\hline & 7 & 0 & 0 & 0 & 0 & 1 & 1 & 0 & 0 & 1 & 1 & 1 & 1 \\
\hline & 8 & 0 & 0 & 0 & 0 & 1 & 1 & 0 & 0 & 1 & 2 & 1 & 2 \\
\hline & 9 & 0 & 0 & 0 & 0 & 1 & 1 & 0 & 0 & 1 & 1 & 1 & 1 \\
\hline & 10 & 0 & 0 & 0 & 0 & 1 & 1 & 0 & 0 & 0 & 0 & 1 & 1 \\
\hline & 11 & 0 & 0 & 0 & 0 & 1 & 1 & 0 & 0 & 0 & 0 & 1 & 1 \\
\hline & 12 & 0 & 0 & 0 & 0 & 1 & 1 & 0 & 0 & 1 & 2 & 1 & 2 \\
\hline & 13 & 1 & 1 & 1 & 1 & 1 & 1 & 2 & 1 & 1 & 2 & 2 & 2 \\
\hline
\end{tabular}

\footnotetext{
* According to T2 criteria.
}

$\dagger$ According to TOF criteria.

For the control group, flow voids in bilateral basal ganglia on T2-weighted images were observed in only 1 control individual (Control 13), with 1 flow void in each side, which were revealed by both 3.0-T and 7.0-T MRI. Because no artery stenosis or occlusion was observed on MRA, the diagnostic category was 1 for all controls for both 3.0-T and 7.0-T MR images.

\section{TOF Criteria}

Similarly, as shown in Table 4, for patients with MMD, 0 or 1 high-signal-intensity area was observed in 14 (46.7\%) sides on 3.0-T and only 1 (3.3\%) side on 7.0-T TOF MRA source images. The number of high-signal-intensity areas detected in 7.0-T TOF source images was significantly greater than that of 3.0-T TOF MRA source images (Fig. $3)$ on both the left ( $p<0.001$, by paired t-test) and right $(\mathrm{p}<0.001$, by paired t-test) sides. According to the TOF criteria, the diagnostic categories were significantly higher in 7.0-T than in 3.0-T MR images ( $\mathrm{p}=0.011$, by Wilcoxon matched-pair signed-rank test).

For the control group, 0 or 1 high-signal-intensity area was observed in 25 (96.2\%) sides on 3.0-T and 21 (80.8\%) sides on 7.0-T TOF MRA source images. The number of high-signal-intensity areas detected in 7.0-T TOF MRA source images was also significantly higher than that of 3.0-T TOF MRA source images on both the left ( $\mathrm{p}=$ 0.014 , by paired t-test) and right $(\mathrm{p}=0.002$, by paired $\mathrm{t}-$ test) sides. The diagnostic categories are shown in Table 4 . Although there were 3 controls in category 2 and 1 control in category 3 according to the 7.0-T MR images, and only 1 control in category 2 according to the 3.0-T MR images, no significant differences were observed between them $(\mathrm{p}=0.102$, by Wilcoxon matched-pair signed-rank test). 
TABLE 5. ICCs between the 2 observers for 3.0-T and 7.0-T MRI/MRA

\begin{tabular}{lcc}
\hline \multicolumn{1}{c}{ Item } & \multicolumn{2}{c}{ ICC Btwn Observers $(95 \%$ CI) } \\
\cline { 2 - 3 } & 3.0-T MRI/MRA & 7.0-T MRI/MRA \\
\hline MRA score & $0.987(0.973-0.994)$ & $0.989(0.978-0.995)$ \\
\hline MMV area score & $0.878(0.755-0.941)$ & $0.977(0.951-0.989)$ \\
\hline MMV quality score & $0.919(0.833-0.961)$ & $0.991(0.981-0.996)$ \\
\hline No. of flow voids in bilat basal ganglia on T2-weighted images & $0.924(0.844-0.964)$ & $0.986(0.970-0.993)$ \\
\hline $\begin{array}{l}\text { No. of high-signal-intensity areas in bilat basal ganglia on source } \\
\text { images of TOF MRA }\end{array}$ & $0.951(0.899-0.977)$ & $0.993(0.985-0.997)$ \\
\hline $\begin{array}{l}\text { Diagnostic category according to T2 criteria } \\
\text { Diagnostic category according to TOF criteria }\end{array}$ & $0.976(0.949-0.989)$ & $0.995(0.990-0.998)$ \\
\hline
\end{tabular}

\section{ICCs for 3.0-T and 7.0-T MRI/MRA}

The ICCs of MRA scores, MMV area scores, MMV quality scores, number of flow voids, number of high-signal-intensity areas, and diagnostic categories between the 2 observers are shown in Table 5; there was good interrater reliability for both 3.0-T and 7.0-T MRI/MRA.

\section{Comparison of STA Visualization Between 3.0-T and 7.0-T MRA}

Both 3.0-T (Fig. 4A) and 7.0-T (Fig. 4B) MRA revealed the main STA and the frontal and parietal branches clearly in all 56 hemispheres. In addition, 7.0-T MRA also revealed the subbranches of the STA in 41 hemispheres, but they could not be seen with 3.0-T MRA.

\section{ROC Curve Analysis of 3.0-T and 7.0-T MRI/MRA}

Figure 5 shows ROC curves for the 2 techniques according to T2 criteria. The AUC of 3.0-T MRI/MRA was 0.800 (95\% CI 0.607-0.926; $\mathrm{p}=0.001$ ). The AUC of the 7.0-T MRI/MRA was 0.992 (95\% CI 0.862-1.000; p < 0.001 ). The difference between the 2 AUCs was 0.192, and a significant difference between the 2 techniques was identified by using Hanlay's method $(\mathrm{p}=0.024)$. In addition, cutoff points were calculated for each technique to maximize sensitivity and specificity using ROC curves. The sensitivity, specificity, and Youden index were 0.692, 0.933 , and 0.626 according to 3.0-T MRI/MRA (calculated cutoff point $>1$ ) and $1.000,0.933$, and 0.933 according to 7.0-T MRI/MRA (cutoff point > 1), respectively.

Figure 6 shows ROC curves for the 2 evaluation meth- ods according to TOF criteria. The AUC was 0.851 (95\% CI 0.666-0.956; $\mathrm{p}<0.001$ ) for the 3.0-T MRI/MRA and 1.000 (95\% CI $0.877-1.000 ; \mathrm{p}<0.001)$ for the $7.0-\mathrm{T}$ MRI/ MRA. The difference between the 2 AUCs was 0.149 , but no significant difference was observed $(\mathrm{p}=0.052)$. Moreover, for the diagnosis of MMD, the sensitivity, specificity, and Youden index were $0.733,0.923$, and 0.656 according to the 3.0-T MRI/MRA (cutoff point > 1) and 1.000, 1.000, and 1.000 according to the 7.0-T MRI/MRA (cutoff point $>3$ ), respectively.

\section{Relationship Between Suzuki's Stage and MRA Grade and Area Score}

For patients with MMD, Spearman's rank correlation test showed a strong correlation between Suzuki's stage and MRA grade in both 3.0-T $\left(\mathrm{r}_{\mathrm{s}}=0.930 ; \mathrm{p}<0.001\right)$ and 7.0-T $\left(r_{s}=0.966 ; p<0.001\right)$ MRA. The correlation between Suzuki's stage and MMV area score was moderate for 3.0-T MRA $\left(\mathrm{r}_{\mathrm{s}}=0.738 ; \mathrm{p}=0.002\right)$ and strong for 7.0-T $\operatorname{MRA}\left(\mathrm{r}_{\mathrm{s}}=0.908 ; \mathrm{p}<0.001\right)$.

\section{Discussion}

MRI and MRA are now widely used for the diagnosis of MMD because of their advantages over conventional DSA, which include noninvasiveness, no requirement for a contrast medium, no radiation exposure, and visual priority for detecting other MR findings (infarction, hemorrhage, and cerebral atrophy), ${ }^{7,26}$ It has been reported that the depiction of small MMVs is better with 3.0-T MRA than with 1.5-T MRA, mainly because the signal-to-noise
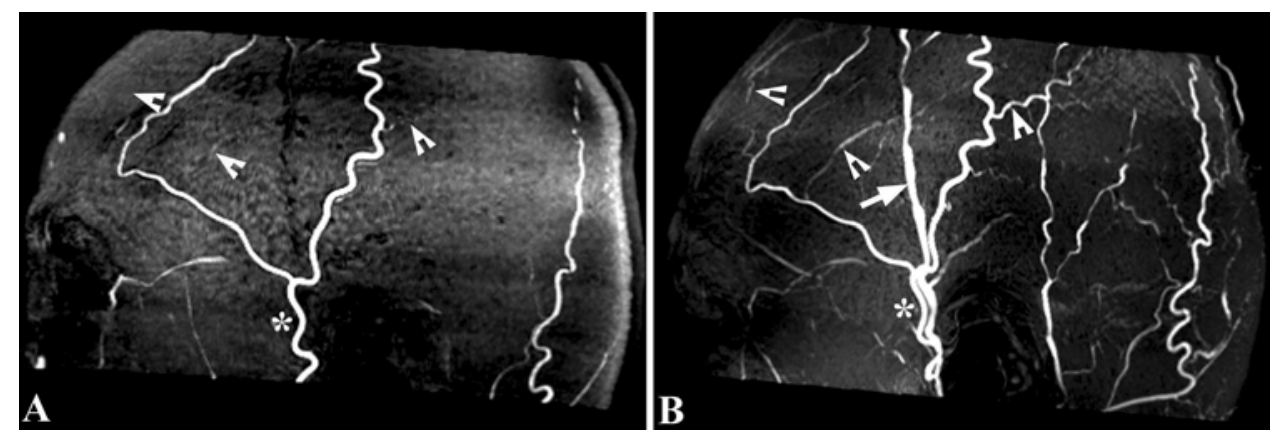

FIG. 4. MIP images of the left STA in lateral view. Both 3.0-T (A) and 7.0-T (B) MRA images delineate the main STA (asterisk) and the frontal and parietal branches clearly. However, 7.0-T MRA also reveals 2 subbranches of the frontal branch, 1 subbranch of the parietal branch (arrowheads), and the superficial temporal vein (arrow), which cannot be seen by 3.0-T MRA. 


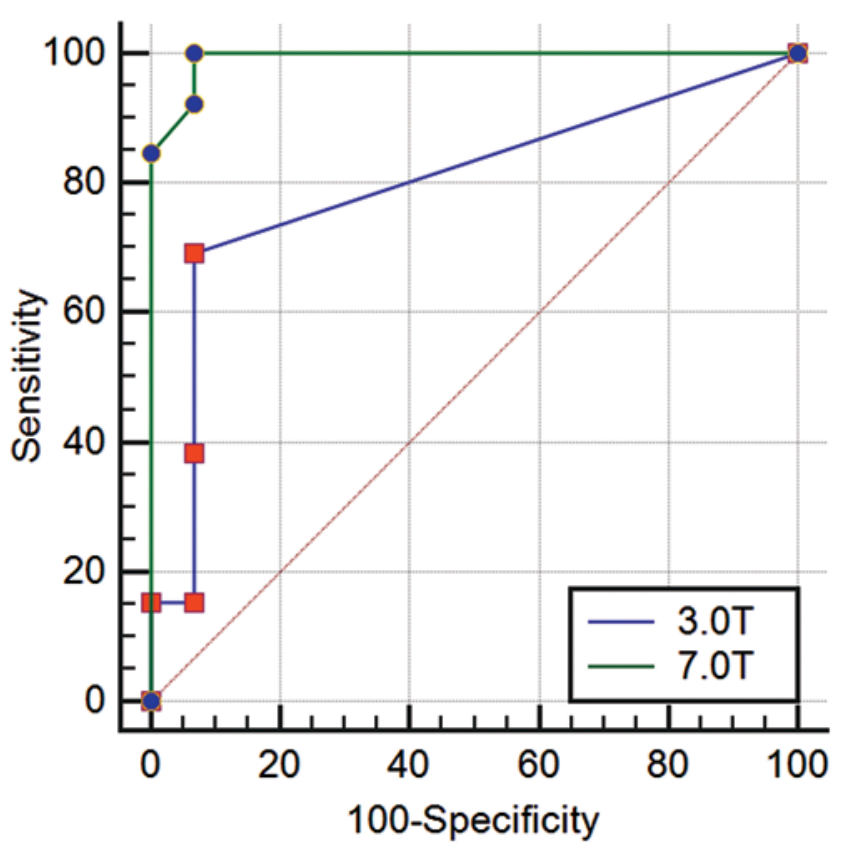

FIG. 5. ROC curves of 3.0-T and 7.0-T MRI/MRA according to T2 criteria. Figure is available in color online only.

ratio of 3.0-T MR images is approximately twice that of 1.5-T MR images. 1,7,33 However, in recent years, 7.0T MRI/MRA has been developed and provides a better signal-to-noise ratio and increased $\mathrm{T} 1$ relaxation time than does 3.0-T imaging, which leads to higher-quality MR images, especially of the small intracranial vessels on TOF MRA. ${ }^{5,28}$ To our knowledge, with the exception of 1 case report, ${ }^{20}$ no other study has focused on comparing 7.0-T and 3.0-T MRI/MRA in patients with MMD. Therefore, we performed this study to compare the image quality of 3.0-T and 7.0-T MRI/MRA, with emphasis on their depiction of MMVs and their sensitivity and specificity for the diagnosis of MMD.

Our results show that there was no significant improvement in the depiction of main intracranial arteries on 7.0-T MRA, as demonstrated by the MRA scores ( $\mathrm{p}=$ $0.317)$ and MRA grades $(p=0.317)$. Both 3.0-T and 7.0-T MRA grades had a strong correlation with Suzuki's stage $\left(r_{s}=0.930\right.$ and 0.966, respectively). Compared with 3.0-T MRA, 7.0-T MRA showed many more small arteries and depicted the MMVs much more clearly, as demonstrated by both the MMV area score $(\mathrm{p}=0.001)$ and MMV quality score $(p=0.001)$. These findings were also consistent with the results of previous studies, which showed that 7.0-T TOF MRA provided only small increases in the signal-to-noise ratio in the primary intracranial vessels but provided better visualization of the first- and second-order branch arteries. ${ }^{11,12,22,36,37}$ Meanwhile, in our study, MMVs in the sylvian fissure, interpeduncular cistern, and quadrigeminal cistern (Fig. 2A and B) were visualized better on axial T2-weighted 7.0-T MR images.

MRA grades according to 3.0-T MRA have been proven to be associated with Suzuki's stage. ${ }^{16}$ Our results correspond with those of previous studies, and 7.0-T MRA did not have a significantly stronger correlation with Suzu-

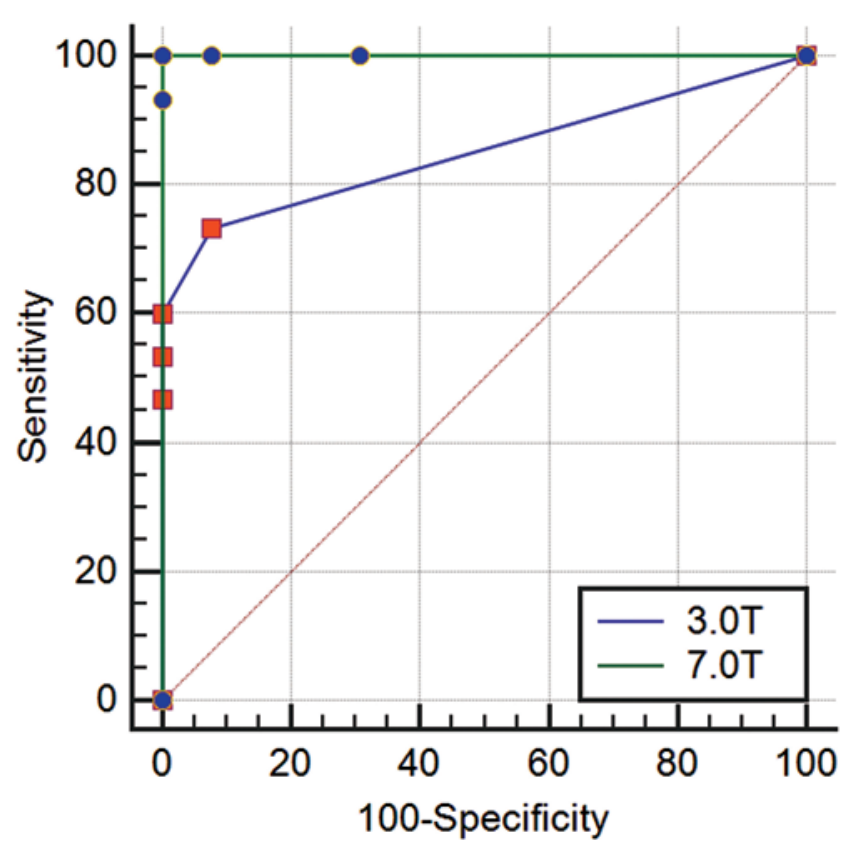

FIG. 6. ROC curves of 3.0-T and 7.0-T MRI/MRA according to TOF criteria. Figure is available in color online only.

ki's stage. The MMV score was excluded from the MRA grade, because the development of MMVs was not significantly correlated with Suzuki's stage. ${ }^{13}$ However, in a study by Jin et al., ${ }^{16}$ there was a good correlation between the MMV score and Suzuki's stage. In our study, the relationship between Suzuki's stage and the MMV area score was moderate for 3.0-T MRA and strong for 7.0-T MRA. We speculate that the probable reason is that the majority (13 of 15) of the patients with MMD were in Suzuki's Stages II to IV, in which the number of MMVs can reflect the severity of the disease.

In addition, visualization of the STA is important, because the STA plays an important role in the surgical treatment of MMD, via either STA-MCA bypass or encephaloduroarteriosynangiosis. Although there was no systematic evaluation of the STA in ultra-high-field TOF MRA, several studies have confirmed better contrast in peripheral segments of intracranial vessels in higher main fields. ${ }^{29,35}$ Our results show that both 3.0-T and 7.0-T MRA can depict the main STA and the frontal and parietal branches clearly. However, compared with 3.0-T MRA, 7.0-T MRA showed a greater ability to detect the tiny branches of the STA; subbranches of the STA on 41 hemispheres were detected by 7.0-T MRA but not by 3.0-T MRA. Therefore, we believe that 7.0-T MRA may provide additional information on the STA, which is useful for surgical treatment.

Regarding the diagnosis of MMD with MRI and MRA, the key point is to detect flow voids in the basal ganglion. ${ }^{2}$ However, previous studies showed that flow voids in the basal ganglion cannot always be observed on MR images, which lowers the diagnostic sensitivity of the criteria. ${ }^{23,27}$ According to the existing guidelines, the sensitivity of 3.0-T MRI/MRA was only 0.45 in the Sawada et al. ${ }^{27}$ study (specificity 1.00 ) and 0.72 in the Mikami et al. ${ }^{23}$ study (specificity 0.74 ). In our study, according to the 
T2 criteria, the diagnostic sensitivity of 3.0-T MRI/MRA was 0.692 (specificity 0.933 ). The low diagnostic sensitivity of 3.0-T MRI/MRA limited its application in the diagnosis of MMD.

In contrast, with respect to 7.0-T MRI/MRA, the diagnostic sensitivity was significantly improved according to both the T2 (sensitivity 1.000; specificity 0.933 ) and TOF (sensitivity 1.000; specificity 1.000) criteria. The high sensitivity and specificity of 7.0-T MRI/MRA might be beneficial for the diagnosis of MMD. If MMD can be diagnosed definitively by using only 7.0-T MRI/MRA, conventional DSA can be skipped, and the adverse effects of DSA, such as contrast media allergy, can be avoided. Moreover, diagnosing MMD by using 7.0-T MRI/MRA alone may be particularly beneficial for younger patients, because DSA usage in pediatric patients may be complicated by narrow arteries and their need for sedation. In addition, it may be beneficial for patients with slow progression, who can be followed up using MRI/MRA alone and treated conservatively without surgical therapy. In conclusion, diagnosing MMD by using 7.0-T MRI/MRA alone may be beneficial for accurate first diagnosis and subsequent imaging follow-ups. Therefore, we speculate that 7.0-T MRI/MRA is a promising technique for diagnosing MMD, because it is noninvasive compared with conventional DSA and has a much higher sensitivity than 3.0-T MRI/MRA.

This study has several limitations. First, it is based on retrospective research with limitations inherent to the study design. Second, the number of patients with MMD was restricted, because only those who underwent DSA and 3.0-T and 7.0-T MRI/MRA were enrolled. Because 7.0-T MRI/MRA is not a routine clinical technique at present, many patients refused to undergo this examination. The small sample size may have led to false-negative findings of the difference of AUCs between the 2 techniques according to the TOF criteria. Third, the parameters of 3.0-T and 7.0-T MRI cannot be matched exactly because of technology limitations and the retrospective design. Moreover, although the readers were blinded to the field strength, there are some differences in image quality between 7.0-T and 3.0-T images. It is possible that the readers were influenced by these differences. In addition, the study was focused on preoperative imaging, and none of these patients underwent postoperative 7.0-T MRI/MRA. Therefore, we could not assess collateralization postoperatively. Last, we cannot deny the possibility of overestimation of steno-occlusion on MRA, as previously reported..$^{15}$

\section{Conclusions}

Compared with 3.0-T MRI and MRA, 7.0-T MR images did not show significant improvement in depicting the major intracranial arteries and the main STA, but it detected and delineated MMVs much more comprehensively, and the tiny branches of the STA were also better visualized. The diagnostic sensitivity was improved significantly by 7.0-T MRI/MRA. We speculate that 7.0-T MRI/MRA is a promising technique for diagnosing MMD because of its noninvasiveness compared with conventional angiography and much higher sensitivity compared with that of 3.0-T MRI/MRA.

\section{Acknowledgment}

This study was supported by the Ministry of Science and Technology of China (Grant 2012CB825505), the National Key Technology Research and Development Program of the Ministry of Science and Technology of China (Grant 2013BAI09B03), and the Center of Stroke, Beijing Institute for Brain Disorders (Grant BIBD-PXM2013_014226_07_000084).

\section{References}

1. Al-Kwifi O, Emery DJ, Wilman AH: Vessel contrast at three Tesla in time-of-flight magnetic resonance angiography of the intracranial and carotid arteries. Magn Reson Imaging 20:181-187, 2002

2. Brady AP, Stack JP, Ennis JT: Moyamoya disease_imaging with magnetic resonance. Clin Radiol 42:138-141, 1990

3. Chiu D, Shedden P, Bratina P, Grotta JC: Clinical features of moyamoya disease in the United States. Stroke 29:13471351,1998

4. Cho ZH, Kang CK, Han JY, Kim SH, Kim KN, Hong SM, et al: Observation of the lenticulostriate arteries in the human brain in vivo using 7.0T MR angiography. Stroke 39:16041606, 2008

5. Dammann P, Barth M, Zhu Y, Maderwald S, Schlamann M, Ladd ME, et al: Susceptibility weighted magnetic resonance imaging of cerebral cavernous malformations: prospects, drawbacks, and first experience at ultra-high field strength (7-Tesla) magnetic resonance imaging. Neurosurg Focus 29(3):E5, 2010

6. Fukui M: Guidelines for the diagnosis and treatment of spontaneous occlusion of the circle of Willis ('moyamoya' disease). Research Committee on Spontaneous Occlusion of the Circle of Willis (Moyamoya Disease) of the Ministry of Health and Welfare, Japan. Clin Neurol Neurosurg 99 (Suppl 2):S238-S240, 1997

7. Fushimi Y, Miki Y, Kikuta K, Okada T, Kanagaki M, Yamamoto A, et al: Comparison of 3.0- and 1.5-T three-dimensional time-of-flight MR angiography in moyamoya disease: preliminary experience. Radiology 239:232-237, 2006

8. Griessenauer CJ, Miller JH, Agee BS, Fisher WS III, Curé JK, Chapman PR, et al: Observer reliability of arteriovenous malformations grading scales using current imaging modalities. J Neurosurg 120:1179-1187, 2014

9. Han C, Feng H, Han YQ, Liu WW, Zhang ZS, Yang WZ, et al: Prospective screening of family members with moyamoya disease patients. PLoS One 9:e88765, 2014

10. Hanley JA, McNeil BJ: A method of comparing the areas under receiver operating characteristic curves derived from the same cases. Radiology 148:839-843, 1983

11. Hendrikse J, Zwanenburg JJ, Visser F, Takahara T, Luijten P: Noninvasive depiction of the lenticulostriate arteries with time-of-flight MR angiography at 7.0 T. Cerebrovasc Dis 26:624-629, 2008

12. Heverhagen JT, Bourekas E, Sammet S, Knopp MV, Schmalbrock P: Time-of-flight magnetic resonance angiography at 7 Tesla. Invest Radiol 43:568-573, 2008

13. Houkin K, Aoki T, Takahashi A, Abe H: Diagnosis of moyamoya disease with magnetic resonance angiography. Stroke 25:2159-2164, 1994

14. Houkin K, Nakayama N, Kuroda S, Nonaka T, Shonai T, Yoshimoto T: Novel magnetic resonance angiography stage grading for moyamoya disease. Cerebrovasc Dis 20:347354,2005

15. Houkin K, Tanaka N, Takahashi A, Kamiyama H, Abe H, Kajii N: Familial occurrence of moyamoya disease. Magnetic resonance angiography as a screening test for high-risk subjects. Childs Nerv Syst 10:421-425, 1994

16. Jin Q, Noguchi T, Irie H, Kawashima M, Nishihara M, Takase Y, et al: Assessment of Moyamoya disease with 3.0-T 
magnetic resonance angiography and magnetic resonance imaging versus conventional angiography. Neurol Med Chir (Tokyo) 51:195-200, 2011

17. Jo KI, Yeon JY, Hong SC, Kim JS: Clinical course of asymptomatic adult moyamoya disease. Cerebrovasc Dis 37:94101,2014

18. Jung MY, Kim YO, Yoon W, Joo SP, Woo YJ: Characteristics of brain magnetic resonance images at symptom onset in children with moyamoya disease. Brain Dev 37:299-306, 2015

19. Kuriyama S, Kusaka Y, Fujimura M, Wakai K, Tamakoshi A, Hashimoto S, et al: Prevalence and clinicoepidemiological features of moyamoya disease in Japan: findings from a nationwide epidemiological survey. Stroke 39:42-47, 2008

20. Lee YB, Kang CK, Kim KT, Park CA, Kim YB, Cho ZH: A non-invasive technique for visualization of microvessels in asymptomatic patients with middle cerebral artery stenoocclusion. Vasc Med 18:314-317, 2013

21. Liu X, Zhang D, Shuo W, Zhao Y, Wang R, Zhao J: Long term outcome after conservative and surgical treatment of haemorrhagic moyamoya disease. J Neurol Neurosurg Psychiatry 84:258-265, 2013

22. Martin-Vaquero P, da Costa RC, Echandi RL, Tosti CL, Knopp MV, Sammet S: Time-of-flight magnetic resonance angiography of the canine brain at 3.0 Tesla and 7.0 Tesla. Am J Vet Res 72:350-356, 2011

23. Mikami T, Sugino T, Ohtaki S, Houkin K, Mikuni N: Diagnosis of moyamoya disease on magnetic resonance imaging: are flow voids in the basal ganglia an essential criterion for definitive diagnosis? J Stroke Cerebrovasc Dis 22:862-868, 2013

24. Noguchi T, Kawashima M, Nishihara M, Hirai T, Matsushima T, Irie H: Arterial spin-labeling MR imaging in Moyamoya disease compared with clinical assessments and other MR imaging findings. Eur J Radiol 82:e840-e847, 2013

25. Park EK, Lee YH, Shim KW, Choi JU, Kim DS: Natural history and progression factors of unilateral moyamoya disease in pediatric patients. Childs Nerv Syst 27:1281-1287, 2011

26. Saeki N, Silva MN, Kubota M, Takanashi J, Sugita K, Nakazaki S, et al: Comparative performance of magnetic resonance angiography and conventional angiography in moyamoya disease. J Clin Neurosci 7:112-115, 2000

27. Sawada T, Yamamoto A, Miki Y, Kikuta K, Okada T, Kanagaki M, et al: Diagnosis of moyamoya disease using 3-T MRI and MRA: value of cisternal moyamoya vessels. Neuroradiology 54:1089-1097, 2012

28. Schlamann M, Maderwald S, Becker W, Kraff O, Theysohn $\mathrm{JM}$, Mueller O, et al: Cerebral cavernous hemangiomas at 7 Tesla: initial experience. Acad Radiol 17:3-6, 2010

29. Schmitter S, Bock M, Johst S, Auerbach EJ, Uğurbil K, Van de Moortele PF: Contrast enhancement in TOF cerebral angiography at $7 \mathrm{~T}$ using saturation and MT pulses under SAR constraints: impact of VERSE and sparse pulses. Magn Reson Med 68:188-197, 2012

30. Scott RM, Smith ER: Moyamoya disease and moyamoya syndrome. N Engl J Med 360:1226-1237, 2009
31. Smith ER, Scott RM: Spontaneous occlusion of the circle of Willis in children: pediatric moyamoya summary with proposed evidence-based practice guidelines. A review. J Neurosurg Pediatr 9:353-360, 2012

32. Suzuki J, Takaku A: Cerebrovascular "moyamoya" disease. Disease showing abnormal net-like vessels in base of brain. Arch Neurol 20:288-299, 1969

33. Thomas SD, Al-Kwifi O, Emery DJ, Wilman AH: Application of magnetization transfer at $3.0 \mathrm{~T}$ in three-dimensional time-of-flight magnetic resonance angiography of the intracranial arteries. J Magn Reson Imaging 15:479-483, 2002

34. Uchino A, Saito N, Takahashi M, Kurita H, Ishihara S: Cerebral arterial variations associated with moyamoya disease diagnosed by MR angiography. Neuroradiol J 27:697-701, 2014

35. von Morze C, Xu D, Purcell DD, Hess CP, Mukherjee P, Saloner D, et al: Intracranial time-of-flight MR angiography at 7T with comparison to 3T. J Magn Reson Imaging 26:900904, 2007

36. Wrede KH, Johst S, Dammann P, Özkan N, Mönninghoff C, Kraemer M, et al: Improved cerebral time-of-flight magnetic resonance angiography at 7 Tesla--feasibility study and preliminary results using optimized venous saturation pulses. PLoS One 9:e106697, 2014

37. Zwanenburg JJ, Hendrikse J, Takahara T, Visser F, Luijten PR: MR angiography of the cerebral perforating arteries with magnetization prepared anatomical reference at $7 \mathrm{~T}$ : comparison with time-of-flight. J Magn Reson Imaging 28:1519-1526, 2008

\section{Disclosure}

The authors report no conflict of interest concerning the materials or methods used in this study or the findings specified in this paper.

\section{Author Contributions}

Conception and design: Zhao, Deng, Z Zhang, R Wang. Acquisition of data: Deng, Z Zhang, B Wang, K Wang. Analysis and interpretation of data: Deng, Ye, Xu, K Wang. Drafting the article: Deng. Critically revising the article: Zhao, Deng, Y Zhang, D Zhang, R Wang, Xu, B Wang, K Wang. Reviewed submitted version of manuscript: Zhao, Deng, Y Zhang, D Zhang, R Wang, $\mathrm{Xu}$, B Wang, K Wang. Approved the final version of the manuscript on behalf of all authors: Zhao. Statistical analysis: Deng, R Wang, Ye, K Wang. Administrative/technical/material support: Z Zhang, Ye, K Wang. Study supervision: Zhao, Z Zhang, Y Zhang.

\section{Correspondence}

Jizong Zhao, Department of Neurosurgery, Beijing Tiantan Hospital, Capital Medical University, No. 6 Tiantan Xili, Chongwen District, Beijing 100050, China. email: zhaojz205@163.com. 